\title{
On the (non-)lattice structure of the equilibrium set in games with strategic substitutes
}

\author{
Sunanda Roy • Tarun Sabarwal
}

Received: 26 December 2006 / Accepted: 24 August 2007 / Published online: 28 September 2007 C Springer-Verlag 2007

\begin{abstract}
This paper studies models where the optimal response functions under consideration are not increasing in endogenous variables, and weakly increasing in exogenous parameters. Such models include games with strategic substitutes, and include cases where additionally, some variables may be strategic complements. The main result here is that the equilibrium set in such models is a non-empty, complete lattice, if, and only if, there is a unique equilibrium. Indeed, for a given parameter value, a pair of distinct equilibria are never comparable. Therefore, with multiple equilibria, some of the established techniques for exhibiting increasing equilibria or computing equilibria that use the largest or smallest equilibrium, or that use the lattice structure of the equilibrium set do not apply to such models. Moreover, there are no ranked equilibria in such models. Additionally, the analysis here implies a new proof and a slight generalization of some existing results. It is shown that when a parameter increases, no new equilibrium is smaller than any old equilibrium. (In particular, in $n$-player games of strategic substitutes with real-valued action spaces, symmetric equilibria increase with the parameter.)
\end{abstract}

Keywords Monotone comparative statics · Non-increasing functions · Never increasing correspondences $\cdot$ Strategic substitutes $\cdot$ Equilibrium set

JEL Classification $\mathrm{C} 60 \cdot \mathrm{C} 61 \cdot \mathrm{C} 62 \cdot \mathrm{C} 72$

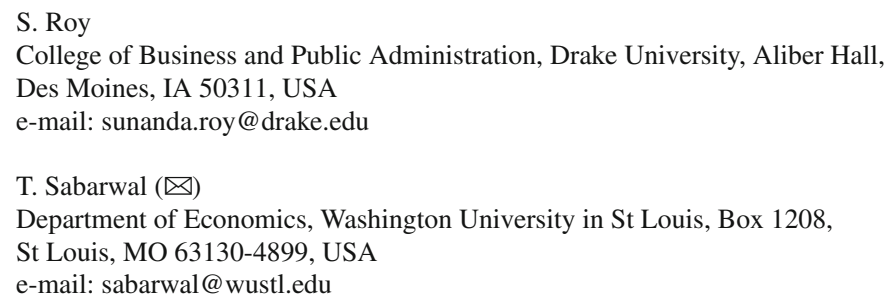




\section{Introduction}

As is well-known, games with strategic complements and strategic substitutes are found in many areas of economics. ${ }^{1}$ Although the structure of the equilibrium set and comparative statics results for general games with strategic complements are welldeveloped, ${ }^{2}$ results of similar generality are less commonly available for games with strategic substitutes, or for games in which the functions under consideration are not increasing in endogenous variables. One result is available for strictly quasi-concave games with strategic substitutes (where best responses of endogenous variables are weakly decreasing functions). As shown by Villas-Boas (1997), in such games, equilibria do not decrease when the exogenous parameter increases. Related earlier papers include Corchón (1994) and Dacic (1981), which explores existence and uniqueness of equilibrium for decreasing maps. ${ }^{3}$

This paper considers models where the product of best response correspondences under consideration are never increasing in endogenous variables, and weakly increasing in exogenous parameters. ${ }^{4}$ Thus, it includes models in which endogenous variables are strategic substitutes for each other, and includes cases where additionally, some endogenous variables may be strategic complements.

The main result shows that for such models, the equilibrium set is a non-empty, complete lattice, if, and only if, there is a unique equilibrium. Indeed, for a given parameter value, a pair of distinct equilibria are never comparable. Therefore, in contrast to games with strategic complements, with multiple equilibria, some of the established techniques for exhibiting increasing equilibria or for computing equilibria that use the largest or smallest equilibrium, or that use the lattice structure of the equilibrium set do not apply to such models. This result also shows that there are no ranked equilibria, and therefore, even with multiple equilibria, these models do not have inefficiencies that arise purely from existence of ranked equilibria. In particular, this result implies

\footnotetext{
1 Such games are defined in Bulow et al. (1985), and as they show, models of strategic investment, entry deterrence, technological innovation, dumping in international trade, natural resource extraction, business portfolio selection, and others can be viewed in a more unifying framework according as the variables under consideration are strategic complements or strategic substitutes. Additional classes of examples are provided by Cournot oligopolies, bargaining games (Nash demand game), and as described in Dubey et al. (2006), games of team projects with complementary or substitutable tasks, and tournaments.

2 Some of this work can be seen in Topkis (1979), Lippman et al. (1987), Sobel (1988), Vives (1990), Milgrom and Roberts (1990), Zhou (1994), Milgrom and Shannon (1994), Milgrom and Roberts (1994), Shannon (1995), Villas-Boas (1997), Edlin and Shannon (1998), Echenique (2002, 2003a, b), and Echenique and Sabarwal (2003), among others. Extensive bibliographies are available in Topkis (1998) and in Vives (1999).

3 Additionally, some aspects of non-monotone mappings that are increasing in some variables and decreasing in others are explored in Roy (2002).

4 As formalized below, a never increasing correspondence is a generalization of a not-increasing function, where a not-increasing function (on a partially ordered set) is defined as $x \prec y \Rightarrow g(x) \npreceq g(y)$. Notice that the term not-increasing is used here to mean the negation of increasing. This also serves to distinguish this term from the case of non-increasing functions, which are sometimes viewed as functions that are decreasing or constant. In particular, not-increasing functions allow for images that are not comparable. A weakly increasing correspondence is a generalization of a weakly increasing function, which is defined, on a partially ordered set, as usual, by $x \preceq y \Rightarrow g(x) \preceq g(y)$.
} 
that in $n$-player games of strategic substitutes with real-valued strategy spaces, if a symmetric equilibrium exists, then it is unique.

Additionally, the analysis here implies a new proof and a slight generalization of some existing results. It generalizes to the case of never increasing correspondences the result by Villas-Boas (1997) for the case of weakly decreasing functions; that is, in such cases, when a parameter increases, no new equilibrium is smaller than any old equilibrium. In the particular case of $n$-player games with strategic substitutes, with real-valued action spaces, symmetric equilibria increase with the parameter.

The paper proceeds as follows. Section 2 presents the main results in three subsections. The first sub-section presents the model, the second sub-section presents the main results on the non-lattice structure of equilibrium sets. The third sub-section applies these results to provide a new proof and a slight generalization of some existing results.

\section{Model and results}

\subsection{Model}

Suppose $(X, \preceq)$ is a partially ordered set, and $A$ and $B$ are subsets of $X$. Then $A$ is weakly smaller than $B$, if for every $a \in A$, there is $b \in B$ such that $a \preceq b$, and for every $b \in B$, there is $a \in A$ such that $a \preceq b$. A correspondence $g: X \rightarrow X$ is weakly increasing, if for every $x, y \in X$ with $x \preceq y$, it is the case that $g(x)$ is weakly smaller than $g(y)$.

A correspondence $g: X \rightarrow X$ is never increasing, if for every $x, y \in X$ with $x \prec y$, for every $x^{\prime} \in g(x)$, and for every $y^{\prime} \in g(y)$, it is the case that $x^{\prime} \npreceq y^{\prime}$. In other words, $g$ is never increasing, if regardless of which point $\left(y^{\prime}\right)$ we choose in the image of a higher point $(y)$, this point is not higher than any point $\left(x^{\prime}\right)$ in the image of a lower point $(x)$. Notice that when $g$ is a function, this definition coincides with that of a not-increasing function; that is, $x \prec y \Rightarrow g(x) \npreceq g(y)$, and moreover, for partially ordered spaces where the partial order is complete, or equivalently, a linear order, ${ }^{5}$ this definition coincides with that of a strictly decreasing function.

Notice that if $g(\cdot, t)$ is a decreasing function, then it is not-increasing. In games of strategic substitutes, the product of best response functions is weakly decreasing. Therefore, the results here apply to games with strategic substitutes. Moreover, notincreasing functions allow for some complementarity as well. An example of a game in which the product of best response functions is not decreasing, but it is not-increasing is given below.

Example 1 Suppose a project has to be completed to reap a reward. ${ }^{6}$ There are three players; players 1 and 2 are capable of completing the project on their own, but player 3 needs to work with either player 1 or player 2 to complete the task. Successful

\footnotetext{
5 To avoid confusion between complete partial orders and complete lattices, this paper uses linear order (a standard term in order theory) to denote a partial order that is complete.

6 This game is based on ideas in Dubey et al. (2006).
} 
players share a parameterized reward $r(t)$ among themselves. ${ }^{7}$ Thus, if only player 1 (or 2) succeeds, he gets $r(t)$ for sure, if players 1 and 2 succeed, (or if players 1 and 3 succeed, or if players 2 and 3 succeed,) each gets $\frac{r(t)}{2}$, and if all three succeed, each gets $\frac{r(t)}{3}$. Each player chooses effort $x_{i} \in[0,1]$, with probability of success $x_{i}$. The quadratic cost of effort is $\frac{c_{i} x_{i}^{2}}{2}$. Expected profit for player 1 is

$$
\begin{aligned}
& r(t) x_{1}\left(1-x_{2}\right)\left(1-x_{3}\right)+\frac{r(t)}{2} x_{1} x_{2}\left(1-x_{3}\right)+\frac{r(t)}{2} x_{1} x_{3}\left(1-x_{2}\right) \\
& +\frac{r(t)}{3} x_{1} x_{2} x_{3}-\frac{c_{1} x_{1}^{2}}{2} .
\end{aligned}
$$

Expected profit for player 2 is given symmetrically, and expected profit for player 3 is

$$
\frac{r(t)}{2} x_{1} x_{3}\left(1-x_{2}\right)+\frac{r(t)}{2} x_{2} x_{3}\left(1-x_{1}\right)+\frac{r(t)}{3} x_{1} x_{2} x_{3}-\frac{c_{3} x_{3}^{2}}{2}
$$

In this case, the best response functions are given as follows.

$$
\begin{aligned}
& \hat{x}_{1}=\min \left\{\frac{r(t)}{c_{1}}\left(1-\frac{1}{2}\left(x_{2}+x_{3}\right)+\frac{1}{3} x_{2} x_{3}\right), 1\right\} \\
& \hat{x}_{2}=\min \left\{\frac{r(t)}{c_{2}}\left(1-\frac{1}{2}\left(x_{1}+x_{3}\right)+\frac{1}{3} x_{1} x_{3}\right), 1\right\} \\
& \hat{x}_{3}=\min \left\{\frac{r(t)}{c_{3}}\left(\frac{1}{2}\left(x_{1}+x_{2}\right)-\frac{2}{3} x_{1} x_{2}\right), 1\right\} .
\end{aligned}
$$

Notice that the joint best response is not decreasing (for small $x_{1}, x_{2}$, player 3's best response is increasing), but it is not-increasing. Moreover, for each $t$, the joint best response is a continuous function on $[0,1]^{3}$, and therefore, a fixed point exists.

The model space for endogenous variables is assumed to be a non-empty, compact, convex subset of Euclidean space, denoted $X$, with the usual product order. The space for exogenous parameters is assumed to be a partially ordered set, denoted $T$. An admissible family of correspondences is a correspondence $g: X \times T \rightarrow X$ such that for every $t$, the correspondence $g(\cdot, t)$ is never increasing, non-empty val-

\footnotetext{
7 For example, the task might be getting a paper published, and two potential authors of the paper can either write the paper by themselves, or jointly, and there is a research assistant who can help complete the paper, but not write the paper herself. If the paper is published then the reward (perhaps some increase in lifetime earnings) is shared among the author(s) and/or research assistant who participated. Or, for example, two potential entrepreneurs are considering offering a new product, and can use the help of another person who does not have the skill to develop and sell the product on his own. If the product is sold, profit is shared among project participants. Other interpretations are possible as well.
} 
ued, compact-valued, convex-valued, and upper hemi-continuous, and for every $x$, the correspondence $g(x, \cdot)$ is weakly increasing. ${ }^{8}$

Consider an admissible family of correspondences $g$, and define the following sets. Let $\underline{\mathrm{S}}(t)=\left\{x \in X \mid \exists x^{\prime} \in g(x, t), x^{\prime} \preceq x\right\}$, let $\bar{S}(t)=\left\{x \in X \mid \exists x^{\prime} \in g(x, t), x \preceq x^{\prime}\right\}$, let $\min \underline{S}(t)$ be the minimal elements of $\underline{S}(t)$, let $\max \bar{S}(t)$ be the maximal elements of $\bar{S}(t)$, and let $F P(t)=\{x \in X \mid x \in g(x, t)\}$ be the fixed points of $g$ at $t$. Kakutani's theorem implies that for every $t, F P(t)$ is non-empty. ${ }^{9}$

\subsection{Non-lattice equilibrium sets}

It is useful to consider one particular reason for the failure of a standard proof of Tarski's theorem when correspondences are never increasing. ${ }^{10}$ This particular failure is notable, because it is related to a modification that does apply in the model considered here, and this modification helps understand the structure of equilibrium sets and non-comparability of equilibria.

In a standard proof, the set $\underline{S}(t)$ has an infimum, $\inf \underline{S}(t) \in \underline{S}(t)$, and inf $\underline{S}(t)$ is the smallest fixed point. Similarly, $\bar{S}(t)$ has a supremum, $\sup \bar{S}(t) \in \bar{S}(t)$, and $\sup \bar{S}(t)$ is the largest fixed point. Monotone increasing selections can then be exhibited by considering these extremal fixed points. With never increasing correspondences, it is easily possible that the set $\underline{S}(t)$ does not contain an infimum, and the set $\bar{S}(t)$ does not contain a supremum. In such cases, the supremum and infimum cannot be fixed points. For example, consider Fig. 1, which gives best response functions of two agents. These functions can be viewed as best responses in a Cournot duopoly where firm one has a lower marginal cost at a higher level of output, and firm two has constant marginal cost. As shown, the product of best responses is a weakly decreasing function. Moreover, $\underline{\mathbf{S}}(t)$ is the area with lower boundary given by ABDE, and it does not contain a smallest point, $\bar{S}(t)$ is the area with upper boundary given by FBCDG, and it does not contain a largest point, $\inf \underline{\mathrm{S}}(t) \notin \underline{\mathrm{S}}(t), \inf \underline{\mathrm{S}}(t) \notin F P(t), \sup \bar{S}(t) \notin \bar{S}(t)$, and $\sup \bar{S}(t) \notin F P(t)$.

Nevertheless, as shown in the following lemma, equilibrium points are minimal elements of $\underline{S}(t)$, and maximal elements of $\bar{S}(t)$. These properties are useful in trying to understand when are equilibria comparable, and when is the equilibrium set a lattice.

Lemma 1 Let $g: X \times T \rightarrow X$ be an admissible family of correspondences. If $x^{*} \in F P(t)$, then $x^{*} \in \min \underline{S}(t) \cap \max \bar{S}(t)$.

Proof Let $x^{*} \in F P(t)$. Then $x^{*} \in g\left(x^{*}, t\right)$, and $x^{*} \preceq x^{*}$, so $x^{*} \in \underline{\mathrm{S}}(t)$. Suppose, by way of contradiction, there is $\hat{x} \in \underline{\mathrm{S}}(t)$ with $\hat{x} \neq x^{*}$, and $\hat{x} \preceq x^{*}$; that is, $\hat{x} \prec x^{*}$. As $\hat{x} \in \underline{S}(t)$, there is $x^{\prime} \in g(\hat{x}, t)$ such that $x^{\prime} \preceq \hat{x}$. In other words, $\hat{x} \prec x^{*}$, and there

\footnotetext{
8 Assumptions other than those regarding correspondences that are never increasing in endogenous variables and weakly increasing in parameters are made to guarantee existence of equilibrium via Kakutani's theorem. Given existence of equilibrium, the results here apply to arbitrary partially ordered $X$.

9 Notice that when $g$ is a family of functions, $\underline{S}(t)=\{x \in X \mid g(x, t) \preceq x\}, \bar{S}(t)=\{x \in X \mid x \preceq g(x, t)\}$, and $F P(t)=\{x \in X \mid x=g(x, t)\}$.

10 For a version of the standard proof, see Topkis (1998, p. 39).
} 


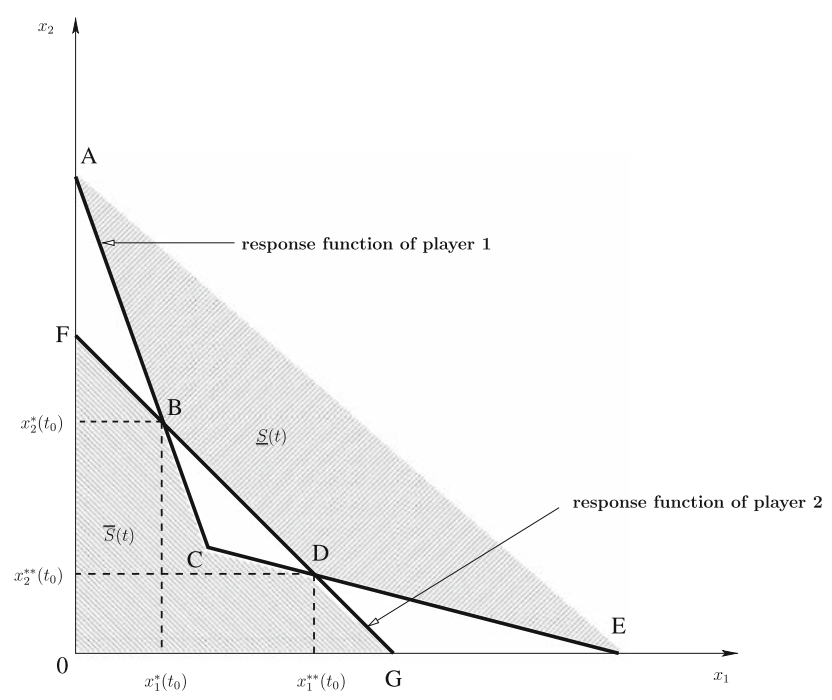

Fig. $1 \max \bar{S}(t), \min \underline{S}(t)$ and non-lattice equilibrium sets

exist $x^{\prime} \in g(\hat{x}, t)$ and $x^{*} \in g\left(x^{*}, t\right)$ such that $x^{\prime} \preceq x^{*}$, contradicting the fact that $g$ is never increasing.

Similarly, $x^{*} \in F P(t) \Rightarrow x^{*} \in \bar{S}(t)$. If $x^{*} \notin \max \bar{S}(t)$, then there is $\hat{x} \in \bar{S}(t)$ such that $x^{*} \prec \hat{x}$. Consequently, there is $x^{\prime} \in g(\hat{x}, t)$, and $x^{*} \in g\left(x^{*}, t\right)$ such that $x^{*} \preceq x^{\prime}$, contradicting the fact that $g$ is never increasing.

In the example provided in Fig. 1, the minimal elements of $\underline{S}(t)$ are given by the boundary depicted by ABDE, and the maximal elements of $\bar{S}(t)$ are given by the boundary depicted by FBCDG, and each of the two equilibria satisfies the conclusion of the lemma. This lemma is useful in proving the following set of results.

Theorem 1 Let $g: X \times T \rightarrow X$ be an admissible family of correspondences. If $x^{*}, \hat{x} \in F P(t)$, and $x^{*} \neq \hat{x}$, then $x^{*}$ and $\hat{x}$ are non-comparable.

Proof The statement follows from the lemma above, as follows. If $x^{*}$ and $\hat{x}$ are distinct fixed points of $g$ at $t$, then these are maximal elements of $\bar{S}(t)$, and hence, these are non-comparable.

Corollary 1 Let $g: X \times T \rightarrow X$ be an admissible family of correspondences. The following are equivalent:

1. $F P(t)$ is a non-empty lattice,

2. $F P(t)$ is a singleton, and

3. $F P(t)$ is a non-empty, complete lattice.

Proof The only part that needs to be checked is that the first statement implies the second. (The other implications are trivial.) Suppose $F P(t)$ is a non-empty lattice, and suppose it contains at least two distinct points, say $x^{*}$ and $\hat{x}$, with $x^{*} \neq \hat{x}$. Then it contains the join and meet of these points, the join and meet are distinct points, and the join and meet are comparable, contradicting the theorem above. 
These results show that for a given parameter value, a pair of distinct equilibria are always non-comparable. In particular, in contrast to equilibria in games with complementarities, this theorem implies that models considered here do not have ranked equilibria. Moreover, as compared to the complete lattice structure of the equilibrium set when correspondences of endogenous variables are increasing (see Zhou 1994), the equilibrium set here is a non-empty, complete lattice exactly in the trivial case of a unique equilibrium. Otherwise, the equilibrium set is totally unordered. Consequently, with multiple equilibria, techniques using the lattice structure of the equilibrium set, or the existence of a smallest and largest equilibrium do not apply to models considered here.

These implications can be seen in Fig. 1, and also in the computed equilibria of a slightly modified version of Example 1. In order to exhibit multiple equilibria for Example 1, suppose the $\operatorname{cost} c_{i}$ for player $i=1,2$ is given by $c_{i}=\bar{c}-\frac{2 c x_{i}}{3}$, where $\bar{c}$ and $c$ are positive constants, and cost for player 3 is the same as in the example. In this case, for a fixed and suppressed value of $t$, and using the paratmeter values $r=1, \bar{c}=0.984, c=0.6$, and $c_{3}=0.48$, three equilibria for this game can be computed to be $(0.906,0.906,0.7474),(0.9957,0.9957,0.6954)$, and $(1,1,0.6944)$. These equilibria are non-comparable. A graphical presentation is given in Fig. 2, where ABC is the best response for players 1 and 2, and DE is the best response of player 3 .

Furthermore, this result implies that in the special case when $X$ is linearly ordered, there is a unique equilibrium for every parameter value. In particular, in $n$-player games of strategic substitutes with real-valued strategies, if a symmetric equilibrium exists, then it is unique.

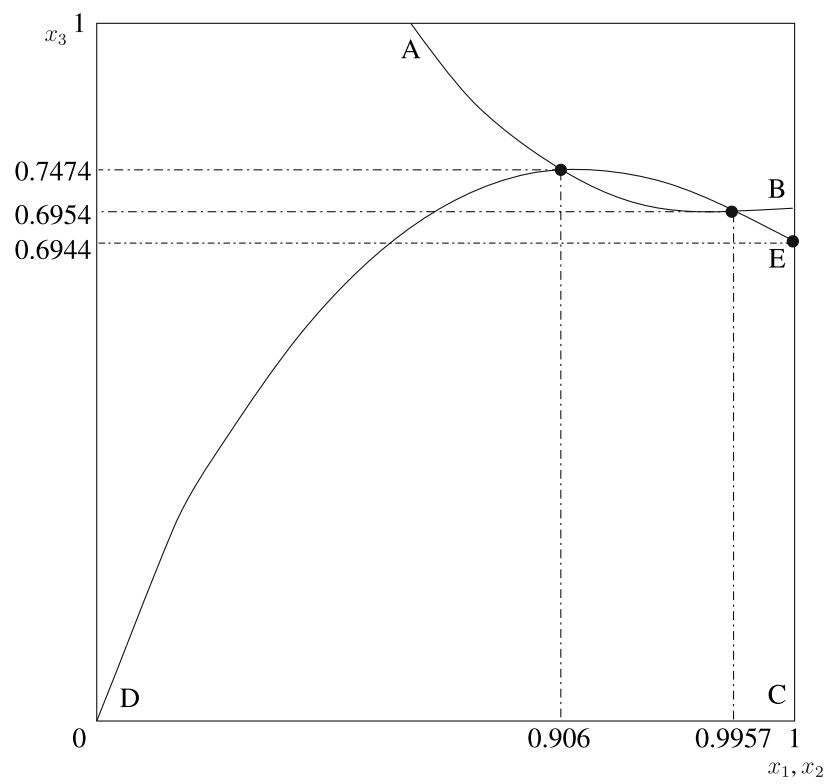

Fig. 2 Non-lattice equilibrium set for Example 1 


\subsection{Nowhere decreasing equilibria}

This sub-section uses results of the previous sub-section to present another proof and a slight generalization of some existing results. The first result generalizes to never increasing correspondences the result by Villas-Boas (1997) for decreasing functions. Its corollary shows that symmetric equilibria are increasing in the parameter.

Theorem 2 Let $g: X \times T \rightarrow X$ be an admissible family of correspondences. For every $t_{1}, t_{2} \in T$, if $t_{1} \preceq t_{2}, x^{*} \in F P\left(t_{1}\right), x^{* *} \in F P\left(t_{2}\right)$, and $x^{*} \neq x^{* *}$, then $x^{* *} \nprec x^{*}$.

Proof When $t_{1}=t_{2}$, the result follows from Theorem 1. Suppose that $t_{1} \prec t_{2}$, and consider distinct fixed points $x^{*} \in F P\left(t_{1}\right), x^{* *} \in F P\left(t_{2}\right)$, and suppose $x^{* *} \prec x^{*}$. Recall that $x^{*} \in F P\left(t_{1}\right) \subset \min \underline{S}\left(t_{1}\right) \subset \underline{S}\left(t_{1}\right)$. Moreover, $g\left(x^{* *}, \cdot\right)$ is weakly increasing in $t$ implies that $g\left(x^{* *}, t_{1}\right)$ is weakly smaller than $g\left(x^{* *}, t_{2}\right)$. As $x^{* *} \in g\left(x^{* *}, t_{2}\right)$, let $x^{\prime} \in g\left(x^{* *}, t_{1}\right)$ such that $x^{\prime} \preceq x^{* *}$. Then $x^{* *} \in \underline{\mathbf{S}}\left(t_{1}\right)$, contradicting the fact that $x^{*}$ is a minimal element of $\underline{\underline{S}}\left(t_{1}\right)$.

This theorem establishes that equilibria are nowhere decreasing in $t$. In particular, this result implies that in the model considered here, there are no decreasing selections of equilibria. Moreover, combined with the previous corollary, it follows that if $X$ is linearly ordered, then there is a unique equilibrium for every $t$, and this equilibrium selection is increasing in $t$. In particular, for games of strategic substitutes with realvalued strategies, symmetric equilibria are increasing, as formalized in the following corollary. $^{11}$

Corollary 2 Consider a game of n-players, each with a non-empty, compact, convex strategy space $X_{i} \subset \Re$, let $X=\times_{i=1}^{n} X_{i}$, T be a parameter space, and $g: X \times T \rightarrow X$ be a product of best response correspondences, and $g$ is never increasing. Say that an equilibrium $x^{*} \in F P(t)$ is a symmetric equilibrium, if for all $i, j, x_{i}^{*}=x_{j}^{*}$. Let $S E(t)$ be the (possibly empty) set of symmetric equilibria. In this case, the following is true. For every $t_{0}, \hat{t} \in T$, if $t_{0} \preceq \hat{t}, x^{*} \in S E\left(t_{0}\right)$, and $x^{* *} \in S E(\hat{t})$, then $x^{*} \preceq x^{* *}$.

Proof If $x^{* *}=x^{*}$, then there is nothing to prove. Otherwise, from the previous theorem, we know that $x^{* *} \nprec x^{*}$. Therefore, there is $i$ such that $x_{i}^{*} \leq x_{i}^{* *}$. As $x^{*}, x^{* *}$ are symmetric equilibria, this implies that $x^{*} \preceq x^{* *}$.

Thus, in this class of games, symmetric equilibria are increasing. Indeed, for this class of games, symmetric equilibria are unique (as shown in the previous sub-section), and therefore (the unique) selection of symmetric equilibria is (weakly) increasing in $t$.

More generally, as is well-known, with asymmetric players, the conclusion of the theorem and corollary above cannot be strengthened to conclude the existence of increasing equilibria, even when the product of best response functions is strictly decreasing in endogenous variable, strictly increasing in parameters, and for every

\footnotetext{
11 As discussed in the introduction, with decreasing best responses, symmetric equilibria can be shown to be increasing using Tarski's intersection point theorem, which requires $X$ to be a chain (see, for example, Milgrom and Roberts 1994). The corollaries here present another proof as an application of the previous theorem, this proof does not require Tarski's intersection point theorem, and it does not require $X$ to be a chain.
} 
parameter value, there is a unique equilibrium. This can be seen through a standard textbook example of a Cournot duopoly with linear demand, constant marginal cost, and an asymmetrically parameterized subsidy of marginal cost.

Notice that the lemma and two theorems in this section apply as stated when $X$ is an arbitrary partially ordered set.

Acknowledgements We are grateful to Federico Echenique, Charles Moul, John Nachbar, Wilhelm Neuefeind, anonymous referees, and seminar audiences at Drake University, Iowa State University, Queen's University, and Washington University in Saint Louis for helpful comments. The results here were circulated earlier as part of the paper, "Monotone Comparative Statics With Never Increasing Correspondencs," RePEc Working Paper Series, No. 0505001, May 2005. A paper by Jensen (2006) with some overlap with our results has been brought to our attention in May 2006. We have exchanged papers with Professor Jensen, and it is our privilege to acknowledge his independent work. An earlier version of the results here was submitted for a round of refereeing in May 2005.

\section{References}

Bulow, J.I., Geanakoplos, J.D., Klemperer, P.D.: Multimarket oligopoly: strategic substitutes and complements. J Polit Econ 93(3), 488-511 (1985)

Corchón, L.: Comparative statics for aggregative games. The strong concavity case. Math Soc Sci 28, 151-165 (1994)

Dacic, R.M.: Properties of monotone mappings in partially ordered sets. Publ De L'Inst Math 30, 33-39 (1981)

Dubey, P., Haimanko, O., Zapechelnyuk, A.: Strategic complements and substitutes, and potential games. Games Econ Behav 54, 77-94 (2006)

Echenique, F.: Comparative statics by adaptive dynamics and the correspondence principle. Econometrica 70(2), 257-289 (2002)

Echenique, F.: The equilibrium set of two player games with complementarities is a sublattice. Econ Theory 22(4), 903-905 (2003a)

Echenique, F.: Mixed equilibria in games of strategic complementarities. Econ Theory 22(1), 33-44 (2003b)

Echenique, F., Sabarwal, T.: Strong comparative statics of equilibria. Games Econ Behav 42(2), 307-314 (2003)

Edlin, A., Shannon, C.: Strict monotonicity in comparative statics. J Econ Theory 81(1), 201-219 (1998)

Jensen M.K.: Existence, comparative statics, and stability in games with strategic substitutes. Working Paper, Department of Economics, University of Birmingham (2006)

Lippman, S.A., Mamer, J.W., McCardle, K.F.: Comparative Statics in non-cooperative games via transfinitely iterated play. J Econ Theory 41(2), 288-303 (1987)

Milgrom, P., Roberts, J.: Rationalizability, learning, and equilibrium in games with strategic complementarities. Econometrica 58(6), 1255-1277 (1990)

Milgrom, P., Roberts, J.: Comparing equilibria. Am Econ Rev 84(3), 441-459 (1994)

Milgrom, P., Shannon, C.: Monotone comparative statics. Econometrica 62(1), 157-180 (1994)

Roy, S.: A note on comparative statics of fixed points of non-monotone mappings. In: Labor Contracts under General Equilibrium: Three Essays on the Comparative Statics of Employment. Ph.D. Dissertation, University of Southern California (2002)

Shannon, C.: Weak and strong monotone comparative statics. Econ Theory 5(2), 209-227 (1995)

Sobel, J.: Isotone comparative statics in supermodular games. Mimeo. SUNY at Stony Brook (1988)

Topkis, D.: Equilibrium points in nonzero-sum $n$-person submodular games. SIAM J Control Optim 17(6), 773-787 (1979)

Topkis, D.: Supermodularity and Complementarity. New Jersey: Princeton University Press, (1998)

Villas-Boas, J.M.: Comparative statics of fixed points. J Econ Theory 73(1), 183-198 (1997)

Vives, X.: Nash equilibrium with strategic complementarities. J Math Econ 19(3), 305-321 (1990)

Vives, X.: Oligopoly Pricing. Cambridge: MIT Press (1999)

Zhou, L.: The set of nash equilibria of a supermodular game is a complete lattice. Games Econ Behav 7(2), 295-300 (1994) 